\section{Descripción de las características del fenómeno Crowding en la Central de Emergencia de Adultos, en un hospital universitario de alta complejidad: estudio de cohorte retrospectiva}

\author{
DIEGO HERNÁN GIUNTA ${ }^{3}$, ANA SOLEDAD PEDRETTI², \\ CRISTINA MARÍA ELIZONDO ${ }^{3}$, MARÍA FLORENCIA GRANDE RATTI ${ }^{3}$, \\ FERNÁN GONZÁLEZBERNALDODE QUIROS ${ }^{3}$, GABRIELDARÍO WAISMAN $^{4}$, \\ HECTOR JOSÉ PERONI ${ }^{1}$, BERNARDO MARTÍNEZ ${ }^{2}$
}

\section{Analysis of Crowding in an Adult Emergency Department of a tertiary university hospital}

Background: Crowding in Emergency Departments (ED), results from the imbalance between the simultaneous demand for health care and the ability of the system to respond. The NEDOCS scale (National Emergency Department Crowding Scale) measures the degree of crowding in an ED. Aim: To describe ED Crowding characteristics, using the NEDOCS scale, in an Argentinean hospital. Material and Methods: A retrospective cohort study was conducted with all adult patient consultations between July 2013 and July 2014 at the ED of Hospital Italiano de Buenos Aires. We included all hours in the analysis period (365 days $\times 24 h=8,760$ ). The NEDOCS value was calculated for each hour using an automatic algorithm and was quantified in a six points score. Levels 4 (overcrowded), 5 (severely overcrowded) and 6 (dangerously overcrowded) were defined as overcrowding. Contour plots analysis was applied to identify patterns. Results: During the study period, 124,758 visits to the ED were registered. Overcrowding was present in $57.7 \%(5,055)$ of the analyzed hours. A predominance of scores between four and five was observed between 10:00 and 24:00 hours. The months with predominance of overcrowding were June, July and August (southern winter). Conclusions: The calculation of the NEDOCS score and the analysis of its temporal distribution are highly relevant to identify opportunities for improvement and to develop mechanisms to prevent the highest categories of overcrowding.

(Rev Med Chile 2017; 145: 557-563)

Key words: Crowding; Emergency Medical Services; Medical Informatics.

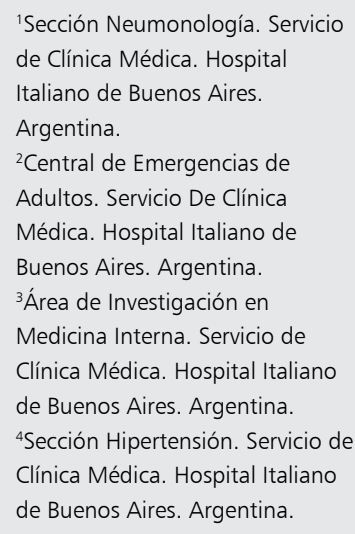

Recibido el 4 de enero de 2016, aceptado el 1 de junio de 2017.

Correspondencia a Dra. María Florencia Grande Ratti Juan D. Perón 4190 (C1181ACH) Buenos Aires, Argentina. maria.grande@hospitalitaliano.org.ar

Anexo en archivo suplementario de la Revista Médica de Chile, en: http://www.scielo.cl
E 1 Crowding -fenómeno de saturación de las Centrales de Emergencias (CE)-, es definido como el desequilibrio entre la demanda simultánea de la atención de salud y la capacidad del sistema para responder. Es consecuencia de un déficit de camas disponibles tanto a nivel hospitalario como en la propia CE. Representa un problema que afecta a la mayoría de los países, independientemente de su nivel socioeconómico, transformándose en un problema de la gestión de todo el hospital y no exclusivo de la $\mathrm{CE}$, por lo que se ha convertido en un problema de salud pública cada vez más importante ${ }^{1,2}$.

El Crowding en la CE dificulta la correcta aten- 
ción del paciente cuando el tiempo de instauración del tratamiento es fundamental, provoca demoras en los diagnósticos y por ende en el inicio de las terapéuticas, se relaciona con un aumento de la morbi-mortalidad, favorece el error humano, e incrementa la estadía hospitalaria, así como los $\operatorname{costos}^{3,4}$.

Uno de los interrogantes más importantes y discutida en la literatura es cómo evaluar el Crowding en la CE, para lo cual se han generado diferentes escalas, de la cuales la más utilizada y que además se encuentra validada en distintos ámbitos de atención de urgencias, incluyendo América Latina, es la escala cuantitativa de NEDOCS (National Emergency Department Overcrowding Scales $)^{5,6}$. Esta herramienta arroja como resultado un valor numérico, permitiendo adicionalmente estratificar en seis categorías el fenómeno.

Hasta el momento no ha sido evaluado el Crowding en nuestro país y la salud no está estructurada del mismo modo aquí que en los lugares donde más se ha estudiado este fenómeno, resulta de gran importancia desde el punto de vista gerencial. El objetivo de este estudio es describir las características del fenómeno a través de la escala de NEDOCS en nuestra institución, un hospital universitario de alta complejidad de la Ciudad Autónoma de Buenos Aires, Argentina.

\section{Material y Método}

Se realizó un estudio de cohorte retrospectiva con el objetivo de describir las características del fenómeno Crowding a través de la escala de NEDOCS en una CE de Argentina. Se utilizaron la totalidad de las consultas a la CE de adultos ocurridas entre 01/07/2013 y 30/06/2014 en la sede central del Hospital Italiano de Buenos Aires (HIBA), Argentina.

El HIBA es un hospital universitario de alta complejidad (tercer nivel), que ofrece servicios médicos integrales en 2 sedes hospitalarias (sede Central y sede de San Justo) y 19 centros médicos ambulatorios periféricos. Atiende cada año 2.950.000 consultas, gestiona 46.000 egresos y realiza 48.500 procedimientos quirúrgicos en sus 41 quirófanos. Cuenta con una capacidad de internación de 750 camas, de las cuales 200 se destinan a cuidados críticos.

Toda la información clínica de los pacientes atendidos (como, por ejemplo, diagnósticos, labo- ratorios, estudios complementarios, evoluciones de consultas ambulatorias, consultas en guardia, internaciones, atenciones no programadas) y administrativa (indicaciones médicas, consumos de farmacia y otros) es recolectada y almacenada en un único repositorio de datos accesible a través de la historia clínica electrónica, que funciona hace más de 10 años. Por ende, la información de la cohorte fue recolectada desde bases de datos secundarias administrativas de alta calidad correspondientes al sistema de atención del HIBA.

En la CE de adultos del HIBA se atienden aproximadamente un promedio de 350 consultas diarias. La unidad de análisis fue cada hora de atención del período del estudio. Se incluyó la totalidad de las horas comprendidas en el período de análisis de un año (365 días por $24 \mathrm{~h}=8.760$ ). El protocolo fue evaluado y aprobado por el Comité de Ética de Protocolos de Investigación institucional (CEPI número 2402).

Como medida de Crowding se utilizó la escala de NEDOCS. Esta herramienta es un instrumento de 5 variables que fue desarrollado en 8 servicios de urgencias de instituciones académicas, y luego validado contra la medición del sobrecupo desde el punto de vista de la carga asistencial del personal médico y de enfermería. Arroja como resultado un valor numérico entre 1 y 200 , y permite clasificar los resultados en seis categorías: (1) 0-20: no ocupado (not busy); (2) 21-60: ocupado (busy); (3) 61-100: muy ocupado (extremely busy); (4) 101-140: hacinamiento (overcrowded); (5) 141180: peligroso (severely overcrowded); (6) > 180: desastre (dangerously overcrowded). Se considera congestión (overcrowding) cuando es mayor a 100, es decir en alguna de las tres últimas categorías: 4 , 5 ó 6 (Figura 1a).

La escala de NEDOCS se encuentra validada en distintos ámbitos de atención de urgencias, incluyendo América Latina ${ }^{5}$. En la estimación de la escala se utilizaron los siguientes componentes según la ecuación que se detalla a continuación ${ }^{7}$ :

$$
\begin{gathered}
\text { NEDOCS }= \\
(-20)+85,8^{\star}(\mathrm{A} 1 / \mathrm{A} 2)+600 *(\mathrm{~B} 1 / \mathrm{B} 2)+ \\
5,64^{\star} \mathrm{C}+0,93{ }^{\star} \mathrm{D}+13,4^{\star} \mathrm{E}
\end{gathered}
$$

(A1) Cantidad pacientes en CE ocupando cama; (A2) Cantidad total de camas en CE;

(B1) Cantidad de pacientes internados en $\mathrm{CE}$ esperando cama de internación; 


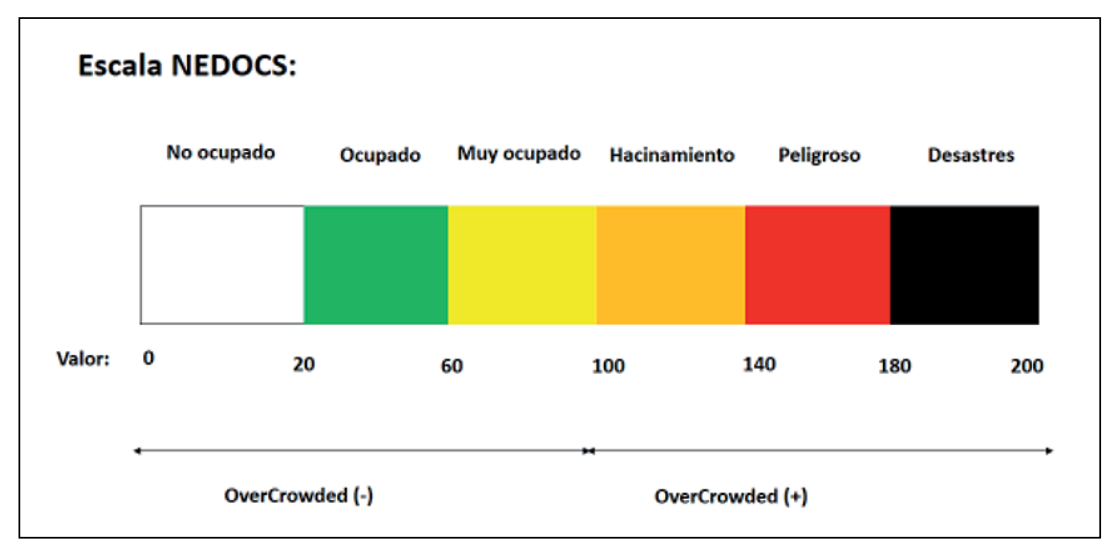

Figura 1a. Diferentes categorías de Crowding según la Escala de NEDOCS.

\section{Tabla 1. Frecuencia relativa de cada categoría de hacinamiento según score de NEDOCS para el período completo}

\begin{tabular}{|l|cc|}
\hline Score NEDOCS & \% & $\begin{array}{c}\text { Frecuencia } \\
\text { absoluta } \\
\text { (total 8.760 h) }\end{array}$ \\
\hline Desastres & 6,6 & 576 \\
\hline Peligroso & 17 & 1.485 \\
\hline Hacinamientos & 34,1 & 2.987 \\
\hline Muy ocupado & 32,3 & 2.830 \\
\hline Ocupado & 10,3 & 902 \\
\hline No ocupado & 0,4 & 40 \\
\hline
\end{tabular}

(B2) Cantidad total de camas del hospital;

(C) Tiempo de espera en sala de espera del último paciente llamado;

(D) El tiempo más largo de espera por cama de internación para pacientes internados en CE;

(E) Cantidad total de pacientes ventilados en CE. Debido a que no fue posible la recolección directa y retrospectiva de la información confiable sobre los pacientes ventilados, se aproximaron los mismos considerando ventilados a aquellos pacientes a quienes se les debitó de farmacia un kit de intubación durante su estadía de atención en la CE (desde la base administrativa de consumos de farmacia utilizada para facturación).

Considerando cada hora como unidad de análisis, se utilizó una herramienta automática que estimó cada una de las cinco variables (A-E) y una medición de Crowding por hora durante el período completo del estudio.

Las variables continuas con distribución normal se expresaron como media y desviación estándar (DS). Las variables categóricas se expresaron como frecuencia absoluta y relativa. Se presentan las figuras con las variaciones de las medidas de Crowding en las diferentes categorías utilizando la escala de NEDOCS, según horas del día, días de la semana y meses del año. Las figuras en función del tiempo se realizaron utilizando Microsoft Office Excel 2007.

Para evidenciar el patrón de Crowding se utilizaron gráficos de contorno. Los gráficos de contornos se utilizan habitualmente para describir profundidad en 2 dimensiones espaciales, con diferentes colores o densidades como $3^{\text {er }}$ dimensión. Se consideraron las $168 \mathrm{~h}$ de la semana $(24 \mathrm{~h}$ por 7 días) en el eje Y, segmentadas cada $24 \mathrm{~h}$, iniciando en la primera hora del lunes. En el eje $\mathrm{X}$ se representaron todas las semanas del año (51 semanas). El color representa el valor de NEDOCS para cada hora representada. En todas las figuras se utilizó el mismo código de color. Los gráficos de contorno y el análisis estadístico se realizaron utilizando STATA versión 14.

\section{Resultados}

Durante el período de estudio se realizaron 124.758 consultas a la CE. La media de edad de los pacientes fue de 49,3 años (DS 21,18), 59,5\% (74.300) fueron de sexo femenino, 45,6\% (56.888) fueron categorizadas al triaje de ingreso con color verde (baja complejidad para su atención), 92,2\% 


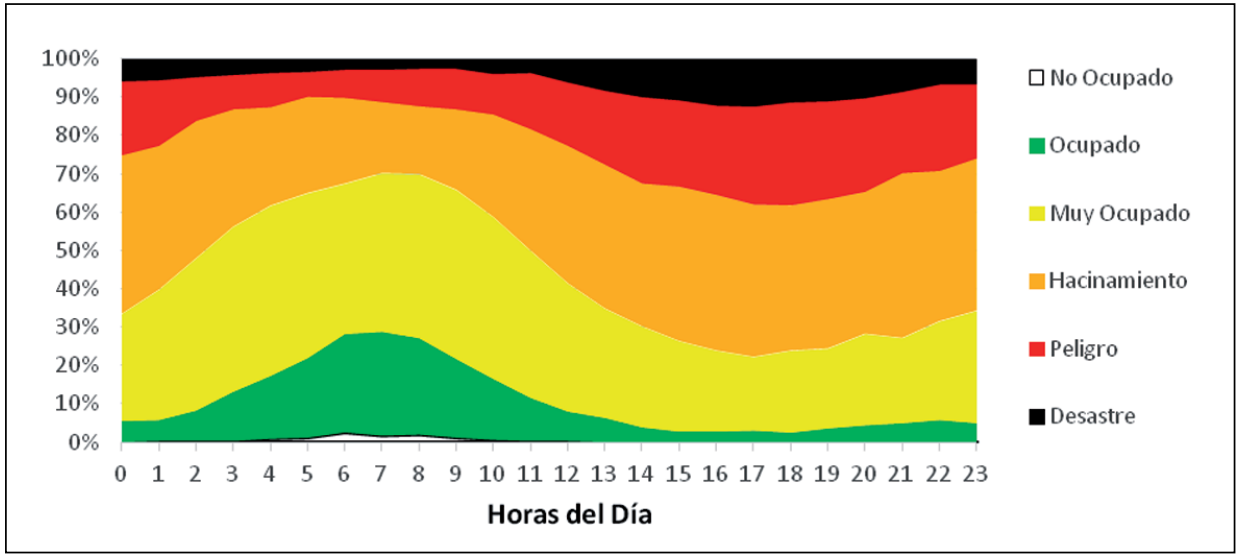

Figura 1b. Porcentaje de Score NEDOCS por horas del día (Horas de 1 a 24).

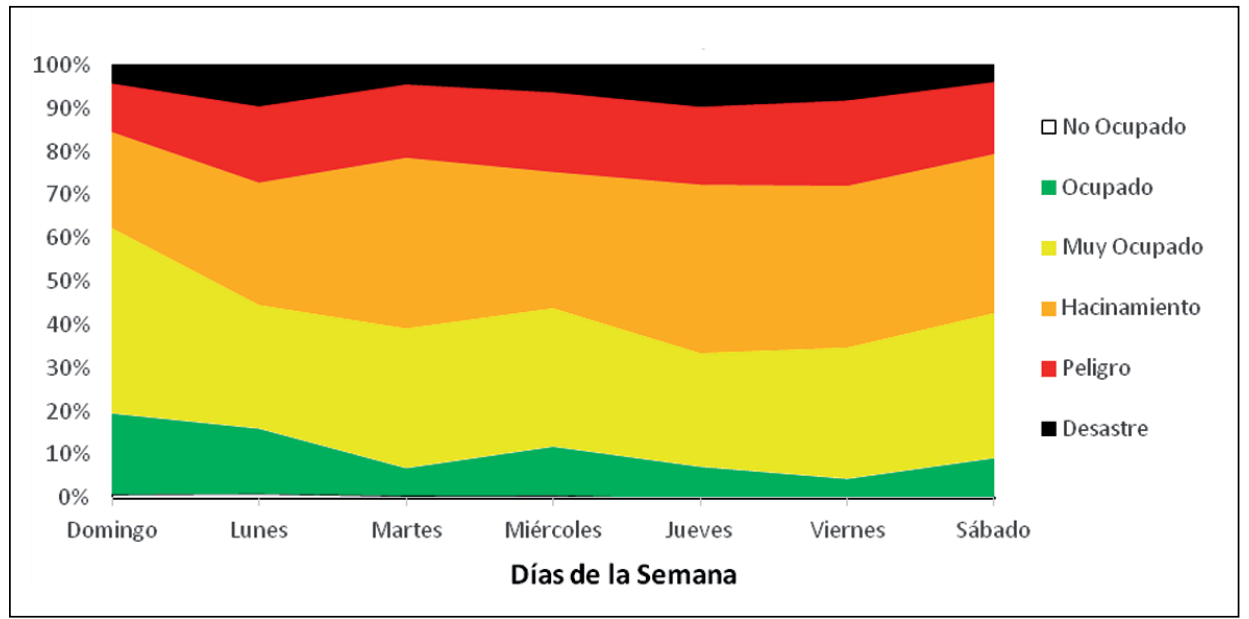

Figura 1c. Porcentaje de Score de NEDOCS por día de la semana.

(115.035) ingresaron caminando por sus propios medios y 10,3\% (12.857) requirieron internación posterior (no programada) como consecuencia de la consulta a la CE.

El total de horas analizadas (8.760) se distribuyeron dentro de los diferentes estratos de la escala de NEDOCS (Tabla 1). El 57,7\% (5.055) de las horas analizadas en la CE permanecieron congestionadas (overcrowded), es decir dentro de las categorías 4, 5 ó 6.

La evaluación de Crowding por horas del día a través de la escala de NEDOCS, permitió detectar que predominan los estratos de hacinamiento y muy ocupado entre las 10:00 y las 24:00 h, presentando un predominio a favor de muy ocupado a partir de este horario hasta las 9:00 h (Figura 1b).

La evaluación de Crowding por días de la semana, permitió detectar que predominan los estratos hacinamiento y muy ocupado. Los días martes, miércoles y jueves presentan un incremento porcentual de los estratos de congestión (overcrowded) (Figura 1c).

La evaluación de Crowding por meses del año, permitió detectar que los estratos hacinamiento y peligroso se incrementan porcentualmente en los meses de junio, julio y agosto. El resto de los meses del año se observó un predominio de los estratos hacinamiento y muy ocupado (Figura 1d).

$\mathrm{Al}$ evaluar el gráfico de contornos para evidenciar el patrón de Crowding global (Figura 2), observamos que durante las horas de la mañana y las primeras de la tarde la CE impresiona encontrarse en los niveles bajos de la escala de NEDOCS (no ocupado, ocupado y muy ocupado); mientras que hacia las horas de la tarde y noche se encuentra en los niveles más elevados de la escala, es decir 


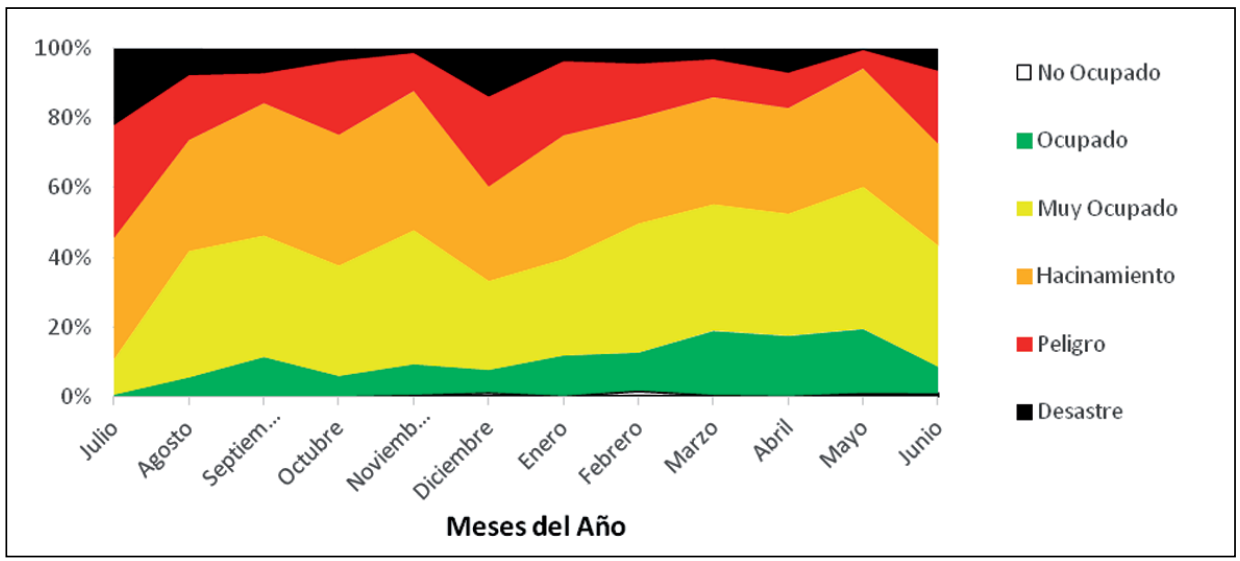

Figura 1d. Porcentaje de Score NEDOCS por meses del año.

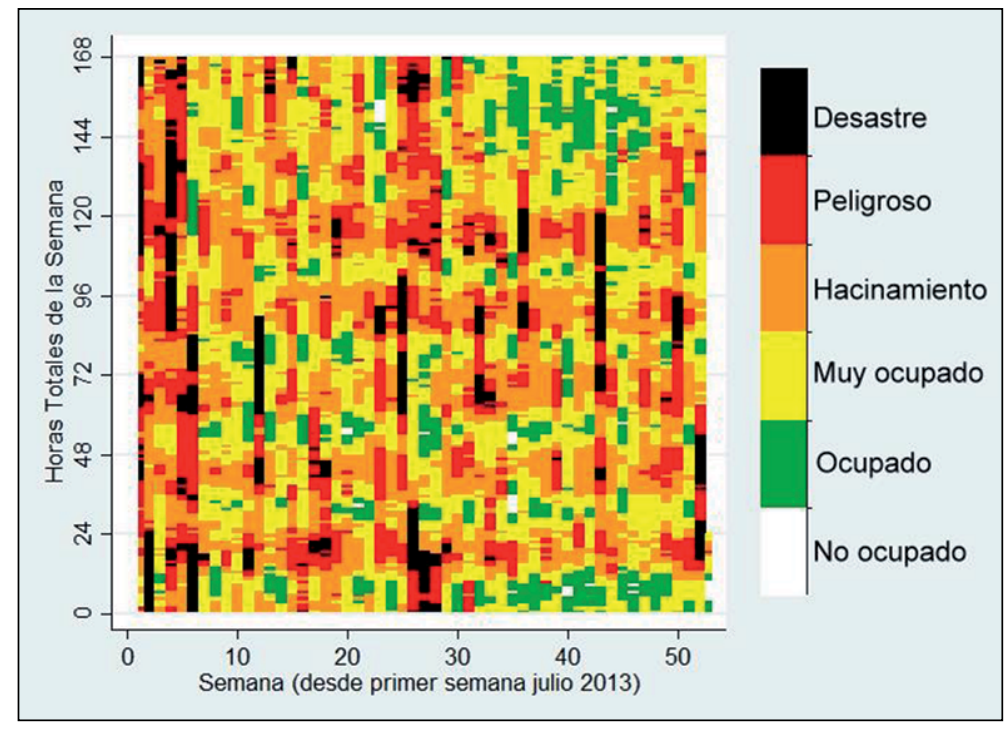

Figura 2. Gráficos de contornos donde el color representa la categoría de NEDOCS. En el eje $X$ se representan todas las semanas del año (51 semanas), en el eje $Y$ se representan las $168 \mathrm{~h}$ de la semana (24 h por 7 días), segmentadas cada $24 \mathrm{~h}$, iniciando en la primera hora del día lunes. congestionada. Adicionalmente, cuando nos detenemos en las semanas 0,30 y 50 se observa un patrón de aumento en la escala de NEDOCS, es decir congestionadas (overcrowded) con respecto al resto de las semanas.

\section{Discusión}

$\mathrm{Al}$ evaluar el fenómeno en la $\mathrm{CE}$, identificamos que la mayor parte de su actividad (57,7\%) se encuentra en los estratos hacinamiento, peligroso o desastre, que llamamos congestionadas (overcrowded). Los motivos que potencialmente explicarían esta situación son: el problema de falta de disponibilidad de camas para la hospitalización de pacientes fuera del área de urgencias; y su consecuencia, la mayoría de los pacientes internados permanecen físicamente en la CE esperando traslado hacia habitaciones, generando el uso permanente de una cama en urgencias destinada a la misma persona durante varios días ${ }^{8}$.

La escala de NEDOCS tiene una precisión diagnóstica apropiada para medir el Crowding en las centrales de emergencias en diferentes poblaciones y ámbitos ${ }^{9,10}$. También se demostró su validez en 
Latinoamérica dado que en Colombia se realizó estudio de validación ${ }^{5}$, por lo que hemos decidido utilizarla y nos permitió obtener una primera aproximación de la situación de la CE de nuestra institución.

Los gráficos de contornos resultaron más útiles que otras herramientas gráficas como aproximación para representar el fenómeno. Al evaluarlos, observamos diferentes conglomerados o patrones en relación al tiempo en horas y días. Este tipo de gráfico nos permite visualizar patrones de comportamiento, los cuales no son posibles de identificar a través de otros tipos de gráficos; y nos permite evidenciar qué comportamiento está teniendo la CE por ejemplo en los meses de invierno, en un determinado momento del día (hora) o en la semana. Representa una herramienta original, que nos permite realizar diagnóstico de situaciones críticas rápidamente, en las cuales podríamos intervenir con el fin de generar ciclos de mejora de gestión.

Dentro de las potenciales limitaciones del estudio nos parece importante destacar que solamente evaluamos la CE de nuestra institución, y esta información, quizás, no pueda extrapolarse al resto de nuestro país o a otros centros de atención de menor complejidad. Sin embargo, es importante resaltar las siguientes fortalezas: representa la primera aproximación en Argentina, y es de gran utilidad intentar explicar este fenómeno, su frecuencia y explorar cómo afecta la atención de la salud en nuestra sociedad. En ese sentido, estudios realizados en otros centros demostraron que el Crowding conlleva categorización inadecuada de los pacientes al ingreso de la CE, demora en el inicio de los tratamientos a instaurar, y mayor mortalidad intrahospitalaria ${ }^{11}$. Debido al gran impacto de estas consecuencias, creemos de vital importancia intentar explicar los patrones que componen este fenómeno, para poder generar herramientas de mejora que nos permitan gestionar de forma más adecuada nuestros recursos. La identificación de los diferentes patrones del grado de Crowding resulta un dato clave, tanto para la gestión del recurso humano como para asegurar la calidad del cuidado ${ }^{12,13}$.

La medición horaria de Crowding a través de la escala de NEDOCS aporta información irremplazable para la toma de decisiones. Las centrales de emergencias están tratando de aliviar el Crowding mediante diversas intervenciones ${ }^{14,15}$. En un futuro, la potencial generación de un sistema que permitiera la medición en tiempo real del grado de Crowding en la CE, podría brinda una herramienta irremplazable para la gestión y por ende, para la seguridad de los pacientes ${ }^{16}$.

Anexo en archivo suplementario de la Revista Médica de Chile, en: http://www.scielo.cl

\section{Referencias}

1. Hoot NR, Aronsky D. Systematic review of emergency department crowding: causes, effects, and solutions. Ann Emerg Med. 2008; 52 (2): 126-36.

2. Anneveld M, van der Linden C, Grootendorst D, Galli-Leslie M. Measuring emergency department crowding in an inner city hospital in The Netherlands. Int J Emerg Med. International Journal of Emergency Medicine 2013; 6 (1): 21.

3. Trzeciak S, Rivers EP. Emergency department overcrowding in the United States: an emerging threat to patient safety and public health. Emerg Med J 2003; 20 (5): 402-6.

4. Arkun A, Briggs WM, Patel S, Datillo PA, Bove J, Birkhahn RH. Emergency Department Crowding: factors influencing flow. West J Emerg Med 2010; 11: 10-5.

5. Amparo J, Canoa C, Olivella EC. Escala Nedocs para medir congestión en Urgencias: 2010.

6. McCarthy ML, Ding R, Pines JM, Zeger SL. Comparison of methods for measuring crowding and its effects on length of stay in the emergency department. Acad Emerg Med 2011; 18 (12): 1269-77.

7. Bair AE, Song WT, Chen YC, Morris BA. The impact of inpatient boarding on ED efficiency: A discrete-event simulation study. J Med Syst 2010; 34 (5): 919-29.

8. Jones SS, Allen TL, Flottemesch TJ, Welch SJ. An independent evaluation of four quantitative emergency department crowding scales. Acad Emerg Med 2006; 13 (11): 1204-11.

9. Weiss S, Derlet R, Arndahl J, Ernst A, Richards J, Fernández $\mathrm{M}$, et al. Estimating the Degree of Emergency Department Overcrowding in Academic Medical Centers: Results of the National ED Overcrowding Study (NEDOCS). Acad Emerg Med 2004; 11: 38-5.

10. Weiss SJ, Ernst AA, Derlet R, King R, Bair A, Nick TG. Relationship between the National ED Overcrowding Scale and the number of patients who leave without being seen in an academic ED. Am J Emerg Med 2005; 23 (3): 288-94.

11. Nathan R, Hoot P, Dominik Aronsky, MD P. Systematic Review of Emergency Department Crowding: Causes, 
Effects, and Solutions. Ann Emerg Med 2008; 52: 12636.

12. Higginson I, Kehoe A, Whyatt J, Smith JE. The 4-hour standard is a meaningful quality indicator: correlation of performance with emergency department crowding. Eur J Emerg Med 2017; 24 (1): 25-8.

13. Khalifa M, Zabani I. Reducing Emergency Department Crowding: Evidence Based Strategies. Stud Health Technol Inform 2016; 226: 67-70.
14. Lauks J, Mramor B, Baumgartl K, Maier H, Nickel CH, Bingisser R. Medical Team Evaluation: Effect on Emergency Department Waiting Time and Length of Stay. PLoS One 2016; 11 (4): e0154372.

15. Crowded EDs leaving proven strategies for improving patient flow on the table. ED Manag 2016; 28 (2): 1922.

16. Carlson K. Crowding in the Emergency Department. J Emerg Nurs 2016; 42 (2): 97-8. 